\title{
MOND as the weak-field limit of an extended metric theory of gravity
}

\author{
S. Mendoza*, T. Bernal* , J. C. Hidalgo* and S. Capozziello ${ }^{\dagger}$ \\ *Instituto de Astronomía, Universidad Nacional Autónoma de México, AP 70-264, Distrito Federal \\ 04510, México. \\ $\dagger$ Dipartimento di Scienze Fisiche, Università degli Studi di Napoli “Federico II”, Complesso \\ Universitario di Monte Sant'Angelo, Edificio N, via Cinthia, 80126, Napoli, Italy \\ I.N.F.N. - Sezione di Napoli, Complesso Universitario di Monte Sant'Angelo, Edificio G, via Cinthia, \\ 80126, Napoli, Italy
}

\begin{abstract}
We show that the Modified Newtonian Dynamics (MOND) regime can be fully recovered as the weak-field limit of a particular theory of gravity formulated in the metric approach. This is possible when Milgrom's acceleration constant is taken as a fundamental quantity which couples to the theory in a very consistent manner. As a consequence, the scale invariance of the gravitational interaction is naturally broken. In this sense, Newtonian gravity is the weak-field limit of general relativity and MOND is the weak-field limit of that particular extended theory of gravity.
\end{abstract}

Keywords: Alternative theories of gravity; modified Newtonian dynamics PACS: $04.50 . \mathrm{Kd}, 04.20 . \mathrm{Fy}, 04.25 . \mathrm{Nx}, 95.30 . \mathrm{Sf}, 98.80 . J \mathrm{~J}, 98.62 . \mathrm{Dm}$

\section{EXTENDED RELATIVISTIC GRAVITY}

As explained by the presentations of Hidalgo and Bernal in this conference series, the inclusion of Milgrom's acceleration constant $a_{0}$ as a fundamental quantity in a description of gravity leads to a great deal of understanding in a wide variety of non-relativistic astrophysical systems. As such, let us assume that a point mass $M$ located at the origin of coordinates generates a relativistic gravitational field in the MONDian regime and that a metric formalism describes the field equations. This problem is characterised by the speed of light in vacuum $c$, the mass $M$ of the central object generating the gravitational field, Newton's constant of gravity $G$ and Milgrom's acceleration constant $a_{0}$. With these parameters, two "fundamental lengths" can be built:

$$
r_{\mathrm{g}}:=G M / c^{2}, \quad l_{M}:=\left(G M / a_{0}\right)^{1 / 2},
$$

where $r_{\mathrm{g}}$ is the gravitational radius and $l_{M}$ is the mass-length scale as described in [1] and by Hidalgo's presentation in this conference series. When relativistic effects are taken into account for the gravitational field, then standard general relativity should be recovered in the limit $l_{M} / r \gg 1$, and a relativistic version of MOND should be obtained when $l_{M} / r \ll 1$. 
This shows that the pursue of a complete metric description leads one to consider the scaledependence of gravity.

The length scales presented in equation (1) must somehow appear in a relativistic theory of gravity which includes the fundamental nature of the constant $a_{0}$. For example, in the metric formalism, a generalised Hilbert action $S_{\mathrm{H}}$ can be written in the following way:

$$
S_{\mathrm{H}}=-\frac{c^{3}}{16 \pi G L_{M}^{2}} \int f(\chi) \sqrt{-g} \mathrm{~d}^{4} x
$$

which slightly differs from its traditional form (see e.g. [2]) since we have introduced the dimensionless Ricci's scalar $\chi:=L_{M}^{2} R$, where $R$ is the standard Ricci's scalar and $L_{M}$ defines a length fixed by the parameters of the theory. The explicit form of the length $L_{M}$ has to be obtained once a certain known limit of the theory is taken, usually a nonrelativistic limit. In fact, since equation (1) defines two natural lengths of the theory it is coherent to postulate the following relation between all these lengths:

$$
L_{M} \propto r_{\mathrm{g}}^{\alpha} l_{M}^{\beta}, \quad \text { with } \quad \alpha+\beta=1 .
$$

Note that the definition of $\chi$ gives a correct dimensional character to the action (2), something that is not completely clear in all previous works dealing with a metric description of the gravitational field. For $f(\chi)=\chi$, the standard Einstein-Hilbert action is obtained. Using the usual form for the matter action $S_{\mathrm{m}}=-(1 / 2 c) \int \mathscr{L}_{\mathrm{m}} \sqrt{-g} \mathrm{~d}^{4} x$, with a matter Lagrangian $\mathscr{L}_{\mathrm{m}}$, then the null variations of the complete action $\delta\left(S_{\mathrm{H}}+S_{\mathrm{m}}\right)=0$ yield the following field equations:

$$
f^{\prime}(\chi) \chi_{\mu \nu}-\frac{1}{2} f(\chi) g_{\mu v}-L_{M}^{2}\left(\nabla_{\mu} \nabla_{v}-g_{\mu \nu} \Delta\right) f^{\prime}(\chi)=\frac{8 \pi G L_{M}^{2}}{c^{4}} T_{\mu \nu}
$$

where the dimensionless Ricci tensor $\chi_{\mu \nu}:=L_{M}^{2} R_{\mu \nu}$ and $R_{\mu \nu}$ is the standard Ricci tensor. The Laplace-Beltrami operator has been written as $\Delta:=\nabla^{\alpha} \nabla_{\alpha}$ and the prime denotes derivative with respect to its argument. The energy-momentum tensor $T_{\mu \nu}$ is defined through the following standard relation: $\delta S_{\mathrm{m}}=-(1 / 2 c) T_{\alpha \beta} \delta g^{\alpha \beta}$. In here and in what follows, we choose a $(+,-,-,-)$ signature for the metric $g_{\mu v}$ and use Einstein's summation convention over repeated indices.

The trace of equation (4) is:

$$
f^{\prime}(\chi) \chi-2 f(\chi)+3 L_{M}^{2} \Delta f^{\prime}(\chi)=\frac{8 \pi G L_{M}^{2}}{c^{4}} T,
$$

where $T:=T_{\alpha}^{\alpha}$. 


\section{MONDIAN WEAK-FIELD LIMIT}

Under the assumption of a power-law relation for the function $f(\chi)$, i.e.

$$
f(\chi)=\chi^{b}
$$

it follows that the trace (5) can be approximated to order of magnitude since $\mathrm{d} / \mathrm{d} \chi \approx 1 / \chi$, $\Delta \approx-1 / r^{2}$ and the mass density $\rho \approx M / r^{3}$ yielding:

$$
\chi^{b}(b-2)-3 b L_{M}^{2} \frac{\chi^{(b-1)}}{r^{2}} \approx \frac{8 \pi G M L_{M}^{2}}{c^{2} r^{3}} .
$$

Note that the second term on the left-hand side of equation (7) is much greater than the first term when $R r^{2} \lesssim 3 b /(2-b)$ and so, since $R \approx \kappa=R_{\mathrm{c}}^{-2}$, where $\kappa$ is the Gaussian curvature of space and $R_{\mathrm{c}}$ its radius of curvature, then $R_{c} \gg r$. This should occur in the weak-field regime, where MONDian effects are expected. For a metric description of gravity, this limit must correspond to the relativistic regime of MOND. In what follows we will only deal with this approximation and so, equation (7) takes the following form:

$$
R^{(b-1)} \approx-\frac{8 \pi G M}{3 b c^{2} r L_{M}^{2(b-1)}} .
$$

Using the fact that at second order of approximation $R=-\left(2 / c^{2}\right) \nabla^{2} \phi=+\left(2 / c^{2}\right) \nabla \cdot \mathbf{a}$, for a non-relativistic potential $\phi$ and an acceleration $\mathbf{a}$, it follows that

$$
a \approx-c^{(2 b-4) /(b-1)} r^{(b-2) /(b-1)} L_{M}^{-2}(G M)^{1 /(b-1)},
$$

which converges to a MOND-like acceleration regime (i.e. $a \propto 1 / r$ ) if $b=3 / 2$. At the lowest order of approximation a description of the gravitational force should not include the velocity of light and so $L_{M} \propto 1 / c$. Combining this result with equation (3) then relation (9) means that

$$
a \approx-\frac{\left(a_{0} G M\right)^{1 / 2}}{r},
$$

which is the traditional form of MOND in spherical symmetry.

As explained by [3] in a more rigorous form, the result obtained in equation (10) is of general character, since it can be formally proved at the lowest order of perturbation for a $f(\chi)=\chi^{3 / 2}$ metric theory of gravity far away from the mass sources, i.e. when $R_{\mathrm{c}} \gg r$, or equivalently when $l_{M} \gg r_{\mathrm{g}}$. In [3] it is also discussed the Noether symmetries of the problem and for this particular case the conserved charge of the problem is proportional to $r_{\mathrm{g}}^{2} l_{M}$

The metric theory of gravity presented here is by no means a complete description at all scales of gravitation. It only deals with the MONDian regime of gravity, i.e. when $l_{M} \gg r_{\mathrm{g}}$ 
is valid. In other words, our description breaks the scale invariance of gravity in a more general way than the one described in [1].

The mass dependence of $\chi$ means that the mass needs to appear on Hilbert's action (2). This is traditionally not the case, since that action is thought to be purely a function of the geometry of space-time due to the presence of mass and energy. However, it was [4] who first encountered this peculiarity in the Hilbert action when dealing with a metric generalisation of MOND and later on also discussed by [5]. Following the remarks of [4], one should not be surprised if some of the commonly accepted notions, even at the fundamental level of the action, require generalisations and re-thinking. An extended metric theory of gravity goes beyond the traditional general relativity ideas and in this way, we probably need to change our standard view of its fundamental principles.

With these ideas in mind it is interesting to note that recently [6] have proposed a modified $F(R, T)$ theory of gravity, where $T$ represents the trace of the energy-momentum tensor. The theory developed in this article is a particular example of their proposal with the identification $F(R, T)=f(\chi) / L_{M}^{2}$. We are developing a cosmological theory with these ideas in mind and we are also applying this full theory to gravitational lenses and to the dynamics of clusters of galaxies. The obtained results will be reported elsewhere.

\section{ACKNOWLEDGEMENTS}

This work was supported by a DGAPA-UNAM grant (PAPIIT IN116210-3). The authors TB, JCH \& SM acknowledge economic support from CONACyT: 207529, 51009, 26344.

\section{REFERENCES}

1. S. Mendoza, X. Hernandez, J. C. Hidalgo and T. Bernal, Mon. Not. Roy. Astron. Soc. 411 (2011) 226

2. S. Capozziello and V. Faraoni, Beyond Einstein Gravity: A Survey of Gravitational Theories for Cosmology and Astrophysics, Springer,(2010) ISBN 9789400701649

3. T. Bernal, S. Capozziello, J.C. Hidalgo and S. Mendoza, European Physical Journal C 71 (2011) 1774

4. Y. Sobouti, Astronomy \& Astrophysics, 464 (2007) 921

5. S. Mendoza and Y. M.Rosas-Guevara, Astronomy \& Astrophysics (2007) 472,367

6. T. Harko, F. S.N. Lobo, S. Nojiri and S. D. Odintsov, Phys.Rev.D (2011) 84:024020 